\title{
Cytotoxic and radiosensitizing effects of FAK targeting in human glioblastoma cells in vitro
}

\author{
KATJA STORCH $^{1 *}$, ANDRE SAGERER $^{1 *}$ and NILS CORDES ${ }^{1,2,3,4}$ \\ ${ }^{1}$ OncoRay - National Center for Radiation Research in Oncology, Faculty of Medicine and University Hospital \\ Carl Gustav Carus, Technische Universität Dresden, and Helmholtz-Zentrum Dresden - Rossendorf, D-01307 Dresden; \\ ${ }^{2}$ Department of Radiation Oncology, University Hospital Carl Gustav Carus, Technische Universität Dresden, D-01307 Dresden; \\ ${ }^{3}$ German Cancer Consortium (DKTK), D-01307 Dresden; German Cancer Research Center (DKFZ), D-69120 Heidelberg; \\ ${ }^{4}$ Institute of Radiooncology, Helmholtz-Zentrum Dresden - Rossendorf, D-01328 Dresden, Germany
}

Received September 25, 2014; Accepted November 27, 2014

DOI: $10.3892 /$ or.2015.3753

\begin{abstract}
Glioblastoma multiforme (GBM) is a highly aggressive and extremely lethal cancer and novel molecular therapies are required for optimized multimodal therapy regimes. While focal adhesion kinase (FAK) is regarded as a therapeutic target, its radiosensitizing potential remains to be elucidated in glioblastoma. Thus, FAK was inhibited using the pharmacological inhibitor TAE226 and cytotoxicity and radiosensitization of glioblastoma cells were investigated in vitro. Monolayer and suspension cell cultures of a panel of glioblastoma cell lines (A172, LN229, U87MG, U138MG, U343MG, DD-HT7607, and DD-T4) were treated with increasing TAE226 concentrations $(0-10 \mu \mathrm{M})$ alone or in combination with X-rays (0-6 Gy). Subsequently, clonogenic cell survival, expression and the phosphorylation of FAK downstream signaling, apoptosis and autophagy were analyzed. Efficient FAK inhibition by TAE22 6 mediated significant cytotoxicity and reduced sphere formation in a dose- and time-dependent manner. Two out of seven glioblastoma cell lines showed radiosensitization. Apoptotic induction by TAE226 was cell line-dependent. The results demonstrated that pharmacological FAK inhibitor TAE226 efficiently reduced clonogenicity and sphere formation in glioblastoma cells without generally modifying their radiosensitivity. However, future studies are necessary to define the
\end{abstract}

Correspondence to: Professor Nils Cordes, OncoRay - National Center for Radiation Research in Oncology, Faculty of Medicine and University Hospital Carl Gustav Carus, Technische Universität Dresden, Fetscherstrasse 74 / PF 41, D-01307 Dresden, Germany

E-mail: nils.cordes@oncoray.de

\section{${ }^{*}$ Contributed equally}

Key words: glioblastoma multiforme, FAK, cytotoxicity, irradiation, cell death potential of FAK inhibition by TAE226 or other pharmacological inhibitors in combination with radiochemotherapy.

\section{Introduction}

Glioblastoma multiforme (GBM) is the most frequent malignant primary brain tumor in adults and is characterized by an aggressive infiltrative growth $(1,2)$. To improve the efficacy of current multimodal therapeutic approaches composed of neurosurgery, radiotherapy and chemotherapy (3), identification of key molecular determinants of radio- and chemoresistance is required. Among the plethora of potential target molecules in GBM, integrins and integrin-associated signaling molecules, such as focal adhesion kinase (FAK), demonstrated promising results in preclinical and early clinical studies (4-8). Peptidomimetic cilengitide, which blocks the RGD binding site of $\alpha v / \beta 3$ and $\alpha v / \beta 5$ integrins $(9,10)$, is the only target strategy that has been previously clinically evaluated. Due to a variety of prosurvival bypass signaling opportunities via other integrin receptors, cilengitide failed to improve overall survival of patients with GBM in Phase IV clinical trials (http://www.merck.com). Despite this drawback, targeting of integrin downstream signaling mediators such as FAK seems reasonable and likely to yield promising results.

FAK is a non-receptor protein-tyrosine kinase that provides signaling and scaffolding functions downstream of integrins. EGFR plays an important role in the regulation of cell processes such as adhesion, survival, migration and invasion $(11,12)$. As FAK is overexpressed in a variety of human tumors including brain, head and neck squamous cell carcinoma (HNSCC), breast, prostate, colon, ovarian, lung, and liver (13-19), targeting of FAK is a promising approach to overcome cancer therapy resistance. Several FAK inhibitors are currently under investigation in clinical trials (http://www. clinicaltrials.gov/ct2/results?term=FAK).

Previously, radiosensitization of human pancreatic and HNSCC was shown by siRNA-mediated FAK knockdown or pharmacological FAK inhibition using TAE226 (20-23). In glioblastoma cells, antisense oligonucleotides against FAK 
enhanced the sensitivity of U251MG cells to chemotherapy such as cisplatin (24). Moreover, TAE226 exhibited a variety of effects in human GBM cells such as delay in proliferation, G2-phase cell cycle arrest, increased apoptosis and attenuated adhesion, migration and invasion (5,7). Of note, studies in intracranial glioblastoma xenograft models showed prolonged survival following TAE226 treatment (5). Due to the fact that patients suffering from GBM received radiotherapy, we assessed the radiosensitizing potential of FAK inhibition using TAE226 in a panel of human glioblastoma cell lines.

\section{Materials and methods}

Antibodies and reagents. Antibodies against FAK Y861 (Abcam, Cambridge, UK), Akt, phospho-Akt S473, phosphoAkt T308, caspase-3, CTTN, phospho-CTTN Y421, cyclin E, ERK1/2, phospho-ERK1/2, FAK, phospho-FAK Y397, IGF-IR, phospho-IGF-IR, JNK, phospho-JNK1/2, LC3-I/II, MEK1/2, phospho-MEK1/2, PARP, Paxillin, phospho-Paxillin Y118, Src, phospho-Src Y118 (Cell Signaling, Danvers, MA, USA), phospho-JNK1/2 (Invitrogen-Life Technologies, Carlsbad, CA, USA), $\beta$-actin (Sigma, St. Louis, MO, USA), cyclin D (Zymed), horseradish peroxidase-conjugated donkey anti-rabbit and sheep anti-mouse (Amersham, Poole, UK) were purchased as indicated. Caspase-3 inhibitor Z-VAD (OMe)-FMK was purchased from Calbiochem (San Diego, CA, USA). The FAK inhibitor TAE226 was kindly provided by Novartis (Cambridge, MA, USA). Enhanced chemiluminescent reagent (ECL) was purchased from Amersham, dimethyl sulfoxide (DMSO) from Applichem (Darmstadt, Germany) and Vectashield/DAPI mounting medium from Alexis (Grünberg, Germany).

Cell culture and X-ray radiation exposure. Human A172, LN229, U87MG, U138MG and U343MG glioblastoma cell lines were obtained from ATCC, while DD-HT7607 and DD-T4 were provided by A. Temme (University Hospital Dresden). The cells were cultured in Dulbecco's modified Eagle's medium (PAA; plus glutamax-I) supplemented with $10 \%$ fetal calf serum (PAA) and $1 \%$ non-essential amino acids (PAA). The U343MG cell line was cultured in Basal Medium Eagle (Life Technologies), supplemented with $10 \%$ fetal calf serum (PAA), $10 \mathrm{mM}$ Hepes, $2 \mathrm{mM}$ L-glutamine, $1 \%$ penicillin/streptomycin (pen/strep) and $1 \%$ non-essential amino acids (PAA) at $37^{\circ} \mathrm{C}$ in a humidified atmosphere containing $7 \% \mathrm{CO}_{2}$. Single doses of $200 \mathrm{kV}$ X-rays $(2,4$ or $6 \mathrm{~Gy}$; Yxlon Y.TU 320; Yxlon; $0.5 \mathrm{~mm}$ copper filter; $1.3 \mathrm{~Gy} / \mathrm{min}, 20 \mathrm{~mA}$ ) were applied at room temperature and measured using a Duplex dosimeter (PTW).

Colony formation assay. Measurement of 2D cell survival was performed as previously published (25). Briefly, single cells were plated in 6-well cell culture dishes (BD Biosciences, Heidelberg, Germany). After $24 \mathrm{~h}$, the cells were treated with indicated amounts of TAE226 or DMSO. In the case of irradiation experiments, TAE226 or DMSO was applied 1,8 or $24 \mathrm{~h}$ prior to irradiation and removed $3 \mathrm{~h}$ after irradiation (single X-ray doses: 2, 4, and 6 Gy). After 9-12 days, depending on the cell line, 2D cell colonies were stained with Coomassie blue
(Merck, Darmstadt, Germany) and counted microscopically. Colonies were defined by cell numbers $>50$. Plating efficiencies were calculated as: Number of colonies formed/number of cells plated. Surviving fractions were calculated as: number of colonies formed/(number of cells plated (irradiated) x plating efficiency (unirradiated). Each point on the survival curves was the mean surviving fraction from at least three independent experiments.

Neurosphere assay. Anchorage-independent growth was enabled using neurobasal medium (PAA), containing $32 \mathrm{U} / \mathrm{ml}$ heparine, $20 \mu \mathrm{l} / \mathrm{ml} \mathrm{B} 27$ supplement, $10 \mu \mathrm{l} / \mathrm{ml}$ glutamax, $20 \mathrm{ng} / \mathrm{ml}$ FGF, $20 \mathrm{ng} / \mathrm{ml}$ EGF, $1 \%$ pen $/ \mathrm{strep}$ and $1 \%$ fungizone (26). The cells were trypsinized and resuspended in neurobasal medium and conferred to plates coated with Ultra-Low attachment surface (Corning, Lowell, MA, USA). After $24 \mathrm{~h}$, the cells were treated with the indicated amounts of TAE226 or DMSO. For the irradiation experiments, TAE226 or DMSO was applied $24 \mathrm{~h}$ prior to irradiation (single X-ray doses: 2, 4, and $6 \mathrm{~Gy}$ ). After 9-12 days, depending on the cell line, the number of spheres with a diameter of $\geq 200 \mu \mathrm{m}$ were counted microscopically. Representative images were captured using an Axioscope 2 microscope (Carl Zeiss, Thornwood, NY, USA) to calculate the sphere size.

DAPI staining. For the apoptosis analysis, the cells were treated at the indicated time points ( 8 or $24 \mathrm{~h}$ ) and fixed with $80 \%$ ethanol for at least $24 \mathrm{~h}$ as previously published (25). Typical apoptotic nuclear shape was analyzed using Vectashield/DAPI mounting medium. Apoptotic nuclei of $\geq 100$ cells from three independent experiments were counted microscopically using an Axioscope 2 microscope (Zeiss).

Total protein extracts and western blotting. Total protein extracts from 2D cell cultures were isolated as previously described (25). Therefore, the cells were lysed using modified RIPA buffer [50 mM Tris-HCl (Carl Roth, Karlsruhe, Germany, (pH, 7.4)], 1\% Nonidet-P40 (Sigma-Aldrich, Taufkirchen, Germany), $0.25 \%$ sodium deoxycholate (Applichem), $150 \mathrm{mM}$ $\mathrm{NaCl}$ (VWR International, Darmstadt, Germany), $1 \mathrm{mM}$ ethylenediaminetetraacetic acid (Merck), complete protease inhibitor cocktail (Roche, Mannheim, Germany), $1 \mathrm{mM}$ $\mathrm{NaVO}_{4}$ (Applichem), $2 \mathrm{mM} \mathrm{NaF}$ (Applichem). The samples were stored at $-80^{\circ} \mathrm{C}$. The total protein amount was measured using the bicinchoninic acid assay (Pierce, Bonn, Germany). The 2D monolayer cells were treated with FAK inhibitor (TAE226, $0.3-10 \mu \mathrm{M}$ ) for 1,8 or $24 \mathrm{~h}$ and subsequently with Caspase 3 inhibitor [Z-VAD (OMe)-FMK, $100 \mu \mathrm{M}$ ] for $1 \mathrm{~h}$ pretreatment, where appropriate.

siRNA-mediated knockown of FAK. FAK siRNA (sense 5'-CCAAAUUCGAGUACUAAGAtt-3') was obtained from Ambion (Austin, TX, USA). A non-specific siRNA (sense 5'-GCAGCUAUAUGAAUGUUGUtt-3'; MWG) was used as the control. The cells were prepared for siRNA transfection $(20 \mathrm{nmol} / \mathrm{l})$ with oligofectamine and were subjected $24 \mathrm{~h}$ after transfection to a colony formation assay and western blotting.

Statistical analysis. Data are presented as the mean \pm SD values. The level of significance was determined by the 
A

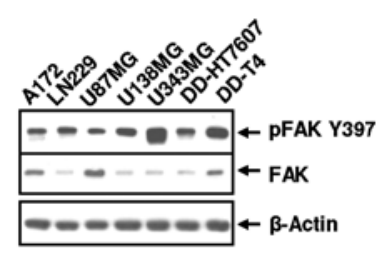

B

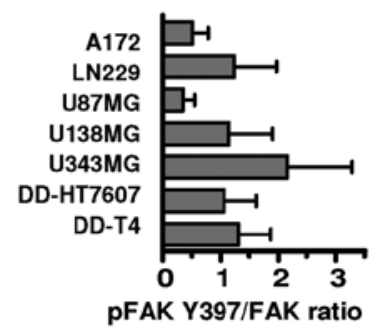

C
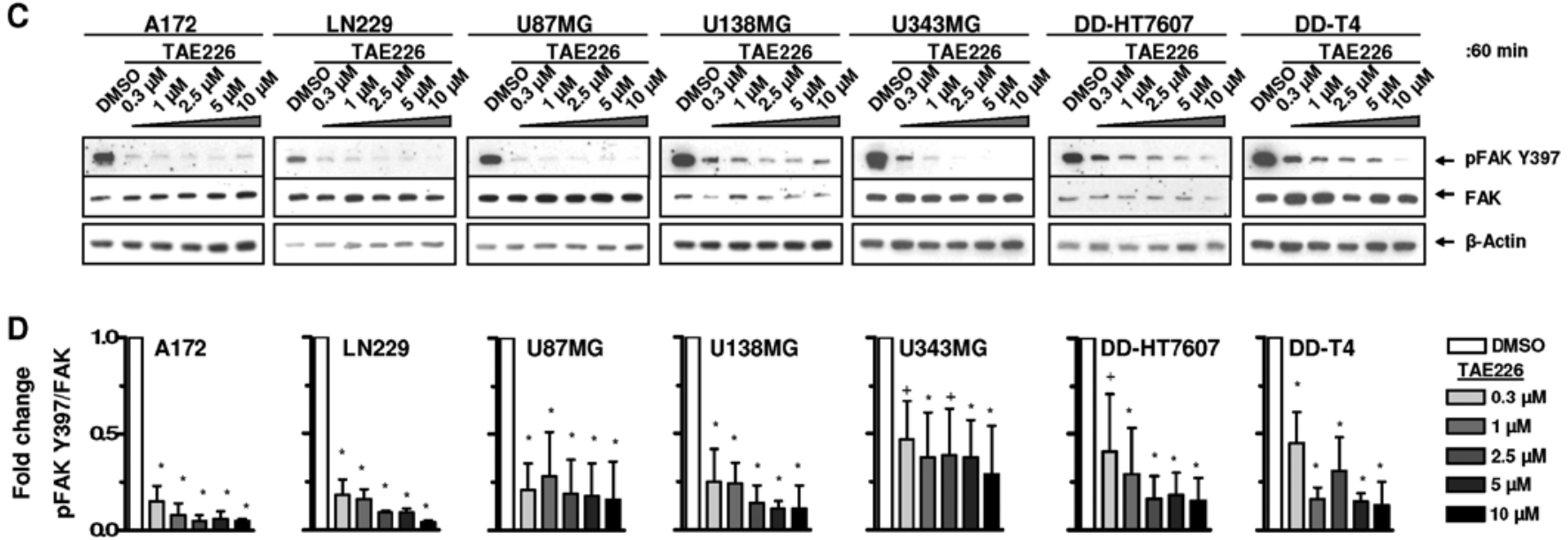

Figure 1. TAE226 significantly reduces FAK Y397 autophosphorylation in glioblastoma cell lines. (A) FAK expression and phosphorylation by western blotting. $\beta$-actin served as the loading control. (B) Densitometric analysis was performed and relative changes in the phosphorylation of FAK (pFAK Y397) were calculated after normalization to the total protein expression. (C) FAK Y397 dephosphorylation efficacy of varying TAE226 concentrations was assessed in 1-h TAE226 treated cells. $\beta$-actin served as the loading control. (D) Densitometric analysis of protein bands shown in (C) was performed and relative changes in pFAK Y397 were calculated from TAE226-treated vs. DMSO-treated cells after normalization to the total protein expression. Results show the mean \pm SD values $\left(\mathrm{n}=3\right.$; $\mathrm{t}$-test; $\left.{ }^{+} \mathrm{P}<0.05,{ }^{*} \mathrm{P}<0.01\right)$

Student's t-test (Microsoft Excel 2010). P $<0.05$ was considered statistically significant.

\section{Results}

TAE226 effectively reduces FAK phosphorylation in human glioblastoma cells. Basal FAK expression and Y397 phosphorylation were examined using a panel of human glioblastoma cell lines (Fig. 1A and B). The inhibitory potential of TAE226 on FAK Y397 autophosphorylation was subsequently assessed (Fig. 1C). TAE226-mediated FAK Y397 dephosphorylation was, although cell line-dependent, not clearly concentration-dependent over the range of 0.3-10 $\mu \mathrm{M}$ (Fig. 1C and D).

TAE226 efficiently reduces FAK autophosphorylation and modulates downstream signaling molecules. We systematically analyzed the potential of TAE226 on various signaling pathways in the LN229, U343MG and DD-T4 glioblastoma cells. FAK and insulin-like growth factor-I receptor (IGF-IR), both reported as targets of TAE226 (27), showed a different response to TAE226 treatment. FAK was dephosphorylated, whereas no modification was detected for IGF-IR (Fig. 2A and B). In the three glioma cells, Paxillin Y118 phosphorylation was reduced by TAE226, while only U343MG and DD-T4 cells revealed reduced phosphorylation of Akt S473/ T308, MEK1/2 and ERK1/2 following TAE226 relative to DMSO (Fig. 2A and B). TAE226 treatment did not affect Src, CTTN and JNK1/2 or the expression of cell cycle proteins Cyclin D or E (Fig. 2A and B).

TAE226 differentially radiosensitizes glioblastoma cells. Cytotoxic effects of TAE226 have been previously reported (4-8). In the present study, we examined the radiosensitizing potential of TAE226 in glioblastoma cells. TAE226 failed to radiosensitize the tested glioma cells when pretreated with $1 \mu \mathrm{M}$ TAE226, whereas $10 \mu \mathrm{M}$ TAE226 pretreatment significantly radiosensitized U138MG and U343MG cells (Fig. 3A and B). Additionally, whether siRNA-mediated FAK targeting provides similar effects, was assessed in U343MG and DD-T4 glioma cells (Fig. 3C). The results showed that U343MG, but not DD-T4, cells were significantly radiosensitized by this approach (Fig. 3D).

TAE226 reduces sphere growth without changing glioblastoma radiosensitivity. TAE226 efficacy in glioblastoma suspension cell cultures in neural basal media was examined. Under these conditions, treatment of single GBM cells with TAE226 led to a shift from larger to smaller spheres with diameters ranging from $>1001$ to $<200 \mu \mathrm{m}$ (Fig. 4A and B). Combined with a 6-Gy single X-ray dose, the frequency of smaller spheres further increased in LN229 and DD-T4 cells (Fig. 4A and B). In line with sphere size, sphere number of LN229 and DD-T4 cells was significantly reduced by 1 or $10 \mu \mathrm{M}$ TAE226 alone (Fig. 4C) while radiosensitization in the GBM cells grown as spheres was lacking (Fig. 4D). Thus, 
A

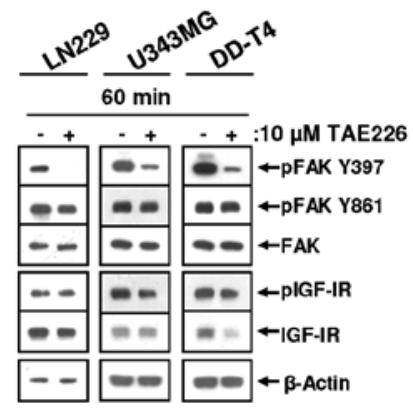

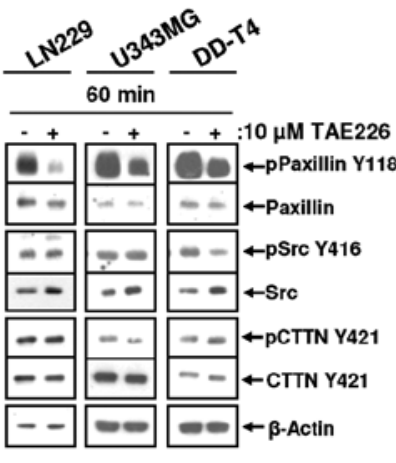

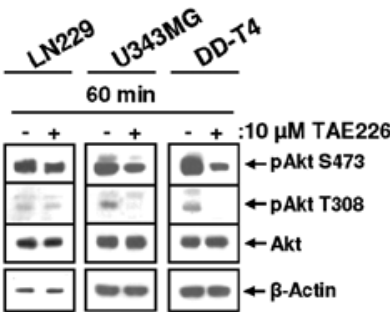

B
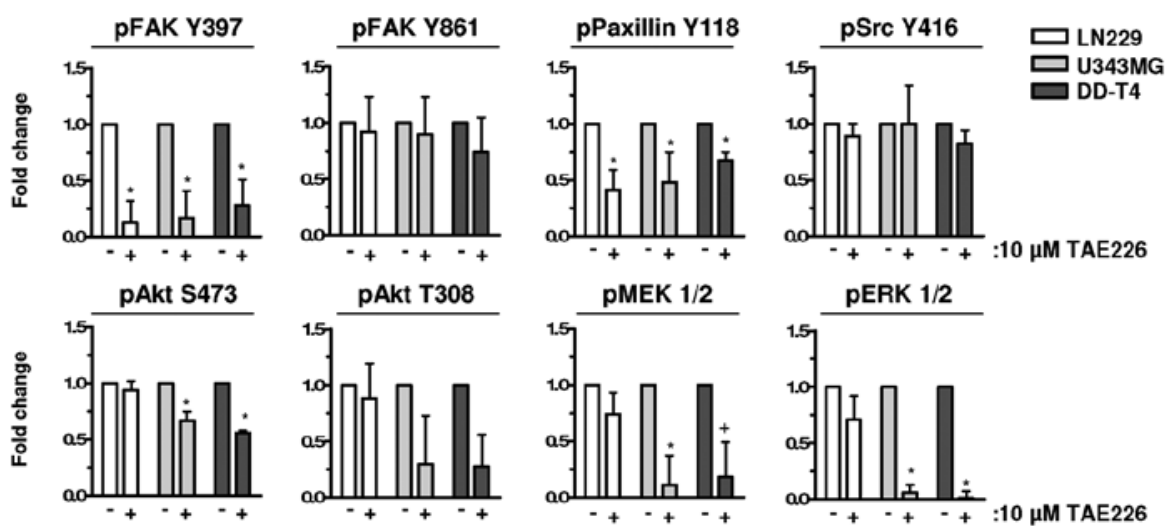

:10 $\mu \mathrm{M}$ TAE226

Figure 2. TAE226 reduces FAK autophosphorylation and modulates downstream signaling molecules in a cell line-dependent manner. (A) Cells were treated with $10 \mu \mathrm{M}$ TAE 226 for $1 \mathrm{~h}$ prior to lysis for total protein extracts and western blot analysis. $\beta$-actin served as the loading control. (B) Densitometric analysis was performed and relative changes in the protein phosphorylation or expression were calculated from TAE226- vs. DMSO-treated cells after normalization to the total protein expression. Results show the mean \pm SD values $\left(\mathrm{n}=3\right.$; t-test; $\left.{ }^{+} \mathrm{P}<0.05,{ }^{*} \mathrm{P}<0.01\right)$.

A

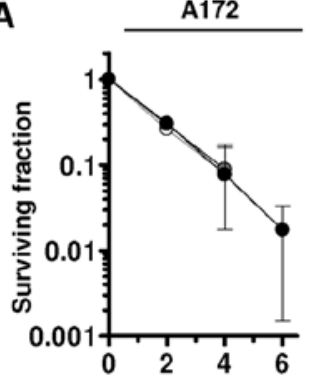

B

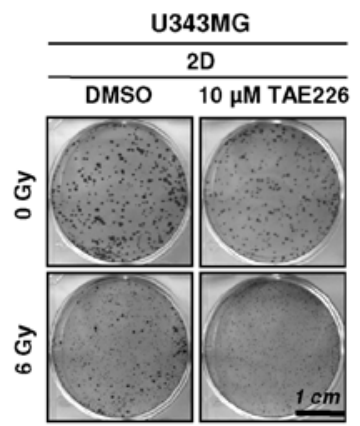

LN229
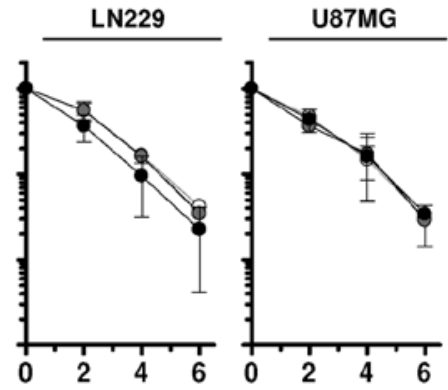

C

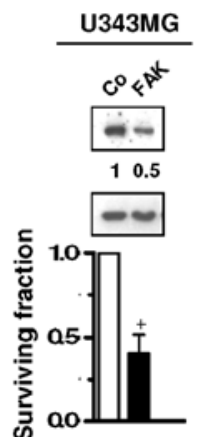

U138MG
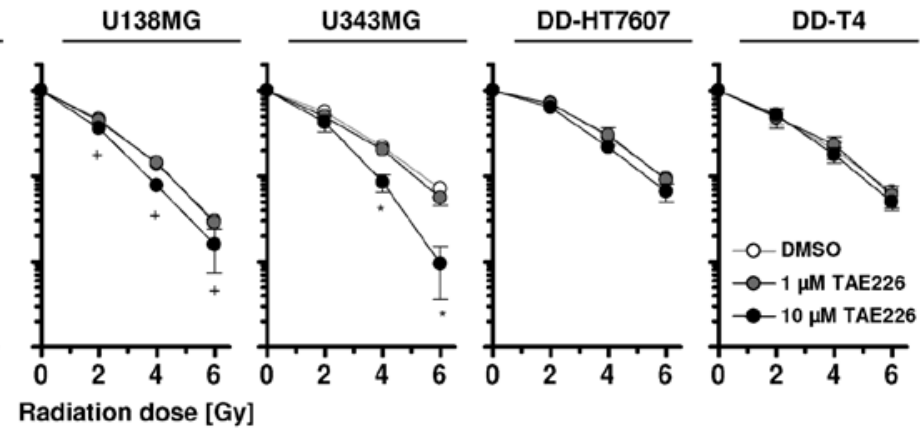

D

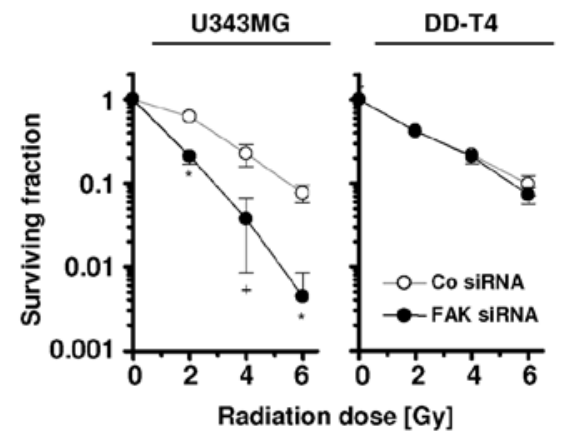

Figure 3. TAE226 confers radiosensitization in a cell line-dependent manner. (A) After a 1-h pretreatment with 1 or $10 \mu \mathrm{M}$ TAE226 or DMSO, the cells were irradiated with X-rays (0-6 Gy) and clonogenicity was determined. (B) Images show colonies of U343MG cells after TAE226 and irradiation. (C) Basal clonogenic survival of U343MG and DD-T4 glioma cells following siRNA-mediated FAK knockdown or non-specific siRNA control. Efficient FAK depletion was confirmed by western blotting. $\beta$-actin served as the loading control. (D) FAK knockdown cell cultures were irradiated (X-ray; 0-6 Gy; single dose) for analysis of radiation survival. Results show the mean $\pm \mathrm{SD}$ values $\left(\mathrm{n}=3\right.$; t-test; $\left.{ }^{+} \mathrm{P}<0.05,{ }^{*} \mathrm{P}<0.01\right)$. Co, control. 

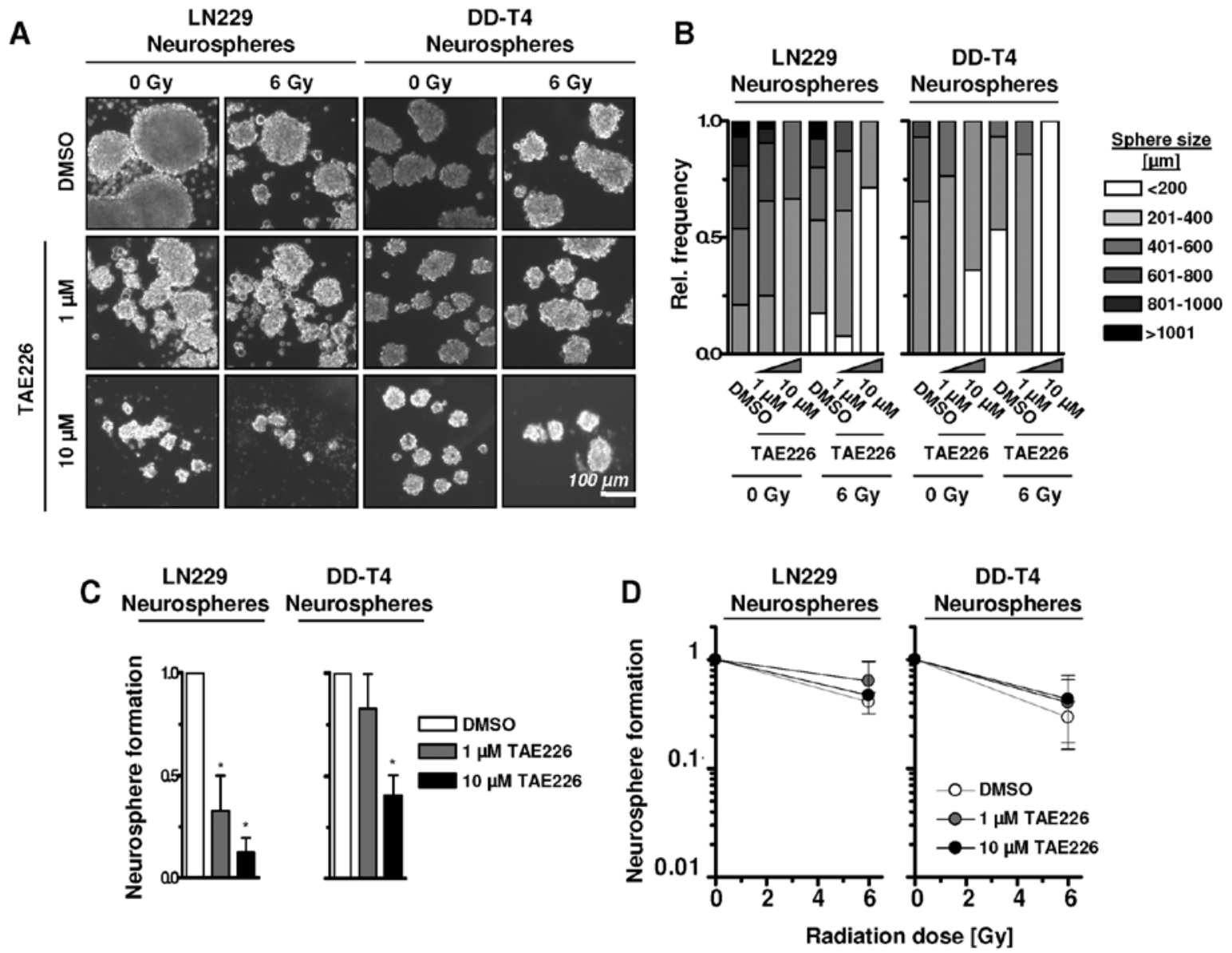

Figure 4. TAE226 reduces neurosphere growth without modifying radiosensitivity. (A) Images show LN229 and DD-T4 neurospheres following TAE226 treatment in combination with X-ray irradiation using phase contrast microscopy. (B) Analysis of neurosphere size upon TAE226 (1 and $10 \mu \mathrm{M}$; DMSO as control) alone or combined with X-ray irradiation. (C) Neurosphere formation of TA226-treated LN229 and DD-T4 cells. (D) Analysis of neurosphere formation following TAE226/irradiation. Results show the mean \pm SD values, $\left(\mathrm{n}=3\right.$; $\mathrm{t}$-test; $\left.{ }^{+} \mathrm{P}<0.05,{ }^{*} \mathrm{P}<0.01\right)$.

under 3D suspension growth conditions, TAE226 has a strong potential for growth inhibition but not for radiosensitization of GBM cells.

TAE226-mediated FAK inhibition induces apoptosis but not autophagic cell death in glioblastoma cells. To assess whether TAE226 induces autophagy and apoptosis in glioblastoma cell lines, we measured LC3-I and LC3-II expression, apoptotic cell nuclei, Caspase 3 and PARP cleavage. In our analysis, LC3-I to LC3-II conversion was not clearly observable following TAE226 indicating autophagy was not an essential cell death process in the examined glioma cells (Fig. 5A). However, TAE226 increased apoptosis relative to DMSO controls in a cell line-, dose- and time-dependent manner (Fig. 5B). The magnitude of apoptosis was not reflected by the degree of cleaved Caspase 3 and PARP (Fig. 5C). By means of the Caspase inhibitor Z-VAD (OMe)-fmk, we showed in U87MG cells with the highest rate of TAE226-induced apoptosis that Caspase 3 cleavage can be completely prevented while the number of apoptotic nuclei is only reduced by $\sim 40 \%$ (Fig. 5D and E). These data suggested apoptosis induction, but not autophagy, contributed to the cytotoxicity of TAE226.

TAE226 strongly attenuates clonogenic cell survival of glioblastoma cells. The impact of TAE226 to reduce clonogenicity of glioblastoma cells was investigated. Increasing TAE226 concentrations significantly reduced the clonogenic survival of all tested glioblastoma cells in a time-dependent manner in comparison to DMSO control.

\section{Discussion}

Optimization of multimodal therapy concepts for GBM is necessary. In this study, we investigated the radiosensitizing and cytotoxic potential of the pharmacological FAK inhibitor TAE226 in glioblastoma cells. We found significant dose- and time-dependent cytotoxicity of TAE226 in a panel of seven human glioblastoma cell lines.

These results are in concordance with previous studies in which TAE226 caused growth inhibition and apoptosis induction in different tumor entities such as glioblastoma (7), neuroblastoma (28), head and neck squamous cell carcinoma (HNSCC) $(21,23)$, esophageal tumors (29) and breast cancer (30). However, monotherapy using a FAK inhibitor is unlikely to occur in the clinic and, thus, knowledge concerning the radiosensitizing potential of TAE226 is of great relevance. TAE226 is described as a potent inhibitor of the tyrosin protein kinases FAK and IGF-IR. Contrary to previously published data $(5,23,27)$, we found significantly reduced FAK Y397 autophosphorylation without modification of IGF-IR 


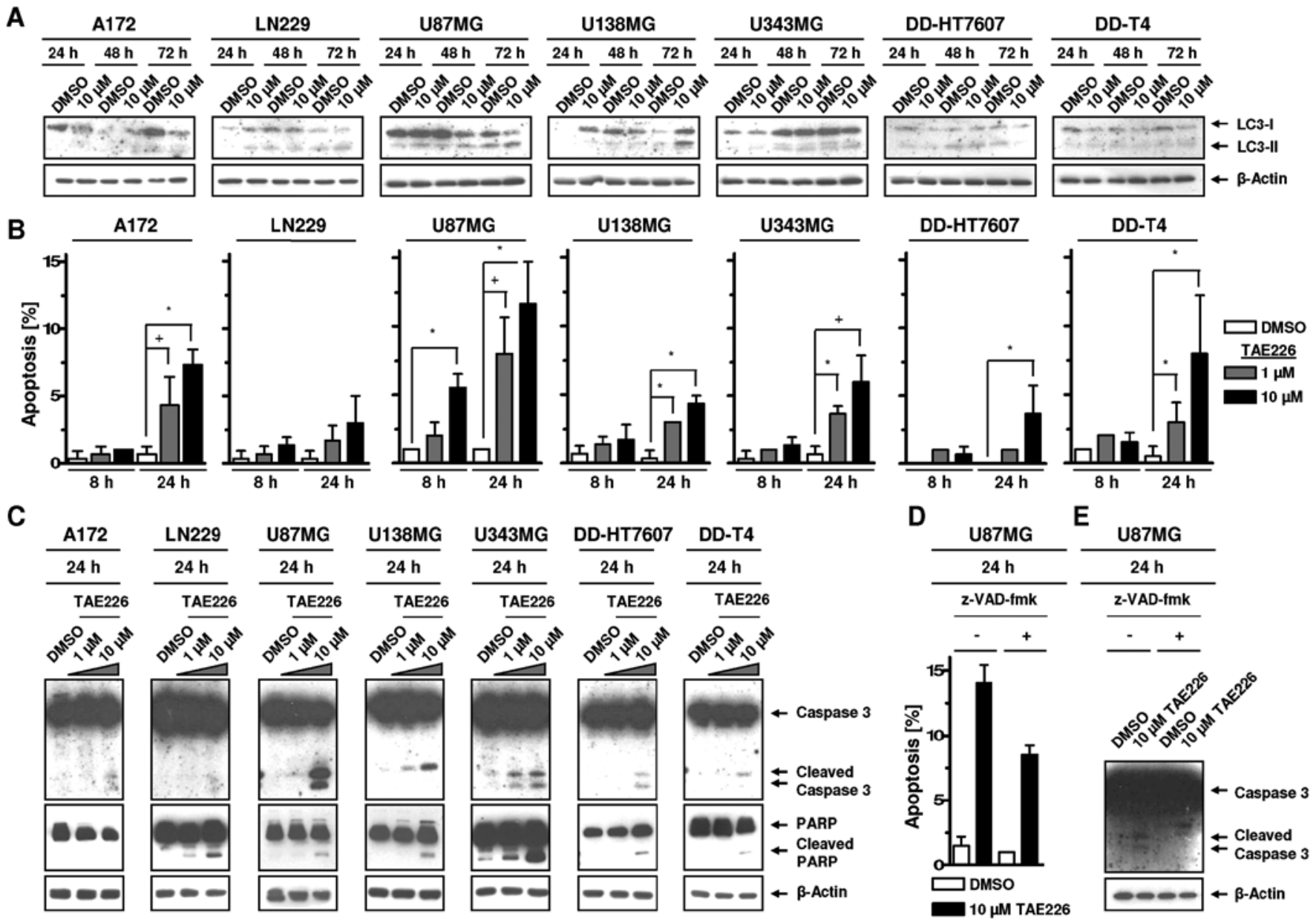

Figure 5. TAE226 induces Caspase 3-dependent apoptosis without affecting autophagy. (A) Glioblastoma cells were treated with $10 \mu \mathrm{M}$ TAE226 for 24 , 48 or $72 \mathrm{~h}$ or DMSO, followed by western blot analysis for LC3-I and LC3-II expression. (B) At the indicated time points, TAE226-treated cells were fixed and stained with DAPI. Cells with apoptotic nuclear morphology were counted to calculate the apoptotic cell fraction. (C) Caspase 3 and PARP expression and cleavage were evaluated under the indicated conditions using western blot analysis. (D) U87MG cells were pretreated with Caspase 3 inhibitor Z-VAD $(\mathrm{OMe})$-fmk prior to TAE226 and stained for apoptosis. Results show the mean \pm SD values $(n=2)$. (E) Western blot analysis of Caspase 3 expression and cleavage of U87MG cells treated with Z-VAD (OMe)-fmk and TAE226 or DMSO. $\beta$-actin served as the loading control. Results show the mean \pm SD values $\left(\mathrm{n}=3\right.$; t -test; $\left.{ }^{+} \mathrm{P}<0.05,{ }^{*} \mathrm{P}<0.01\right)$.
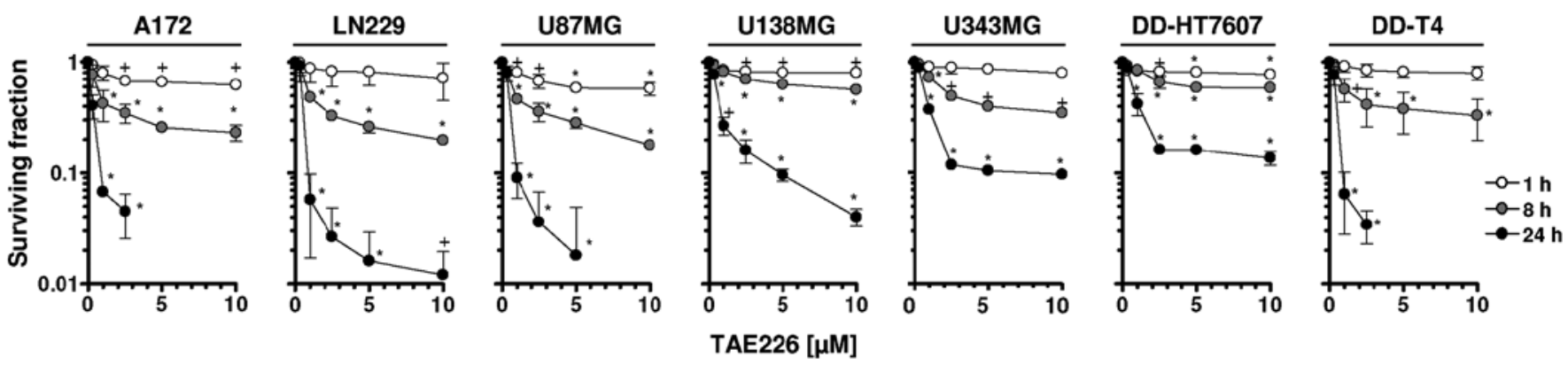

Figure 6. TAE226 reduces GBM cell clonogenicity in a time- and concentration-dependent manner. Cytotoxic effects of $0.3,1,2.5,5$ and $10 \mu \mathrm{M}$ TAE226 incubated over 1,8 or $24 \mathrm{~h}$ relative to DMSO were determined. Results show the mean $\pm \mathrm{SD}$ values $\left(\mathrm{n}=3\right.$; $\mathrm{t}$-test; $\left.{ }^{+} \mathrm{P}<0.05,{ }^{*} \mathrm{P}<0.01\right)$.

phosphorylation following TAE226 application in glioblastoma cells. Similar to other studies $(5,23)$, FAK inhibition led to alterations of adapter and signaling molecules directly or partly associated with FAK such as Paxillin, Akt, MEK1/2 and ERK1/2.
Owing to this multitarget deactivation, TAE226 mediated strong cytotoxicity that may be attributed to apoptosis but not autophagy. Similar data have been published by other authors $(7,24)$. Moreover, this apoptosis induction presented partly Caspase-dependent and partly Caspase-independent, 
thus further mechanistic examination is required. Of note, apoptosis or anoikis is stimulated following TAE226-dependent FAK Y397 autophosphorylation in GBM cells indicating the importance of integrin-mediated anchorage-dependent growth of GBM cells. The broad inhibitory spectrum of TAE226 is beneficial to induced cell death in suspension cell cultures, in which cell-ECM interactions are less relevant for cell survival. Hypothesizing TAE226 to activate different cell death modes under adhesion vs. suspension seems of high interest for the clinical scenario where different GBM cell populations compose the tumor heterogeneity and are likely to be differentially dependent on adhesion.

Based on the fact that small molecules are administered in combination with conventional radiotherapy or radiochemotherapy, we investigated TAE226 plus irradiation and found that only a small number of GBM cell lines are radiosensitized. Previous studies testing TAE226/irradiation showed that head and neck cancer cells, but not carcinoma cells, from lung, colorectum and pancreas can be sensitized to radiotherapy $(21,23)$. Targeting of $\beta 1$ integrin, located upstream of FAK, mediated less cytotoxicity than TAE226 but radiosensitization in human GBM cell lines (4). These observations suggest that: i) GBM cell survival is highly FAK-dependent; ii) the molecular circuitry controlled by targets of TAE226 does not essentially contribute to the DNA damage response of GBM cells.

In conclusion, our findings demonstrate that the pharmacological FAK inhibitor TAE226 efficiently reduces clonogenic survival without radiosensitization in a panel of glioblastoma cell lines. In the future, the identification of the integrin-related adhesome seems to be key for the optimization of therapeutic strategies for GBM as the multiprotein-multifunctional adhesome complexes control prosurvival signaling and invasion processes.

\section{Acknowledgements}

This study was supported by The German Federal Ministry of Education and Research; contract grant number: BMBF Contract 03ZIK041 (to N.C.) and by The Saxon Ministry of Science and Arts and the EFRE Europäische Fonds für regionale Entwicklung, Europa fördert Sachsen; Contract grant no. 100066308 . We are grateful to A. Temme (University Hospital Dresden, Germany) for providing U343MG, DD-HT7607 and DD-T4 cells. The pharmacological FAK inhibitor TAE226 was kindly provided by Novartis Institutes of Biomedical Research, Inc. (Cambridge, MA, USA).

\section{References}

1. Louis DN, Ohgaki H, Wiestler OD, Cavenee WK, Burger PC, Jouvet A, Scheithauer BW and Kleihues P: The 2007 WHO classification of tumours of the central nervous system. Acta Neuropathol 114: 97-109, 2007.

2. Bleeker FE, Molenaar RJ and Leenstra S: Recent advances in the molecular understanding of glioblastoma. J Neurooncol 108: 11-27, 2012.

3. Becker KP and Yu J: Status quo - standard-of-care medical and radiation therapy for glioblastoma. Cancer J 18: 12-19, 2012.

4. Eke I, Storch K, Kastner I, Vehlow A, Faethe C, MuellerKlieser W, Taucher-Scholz G, Temme A, Schackert G and Cordes N: Three-dimensional invasion of human glioblastoma cells remains unchanged by X-ray and carbon ion irradiation in vitro. Int J Radiat Oncol Biol Phys 84: e515-e523, 2012.
5. Liu TJ, LaFortune T, Honda T, Ohmori O, Hatakeyama S, Meyer T, Jackson D, de Groot J and Yung WK: Inhibition of both focal adhesion kinase and insulin-like growth factor-I receptor kinase suppresses glioma proliferation in vitro and in vivo. Mol Cancer Ther 6: 1357-1367, 2007.

6. Rieken S, Habermehl D, Mohr A, Wuerth L, Lindel K, Weber K, Debus J and Combs SE: Targeting $\alpha v \beta 3$ and $\alpha v \beta 5$ inhibits photon-induced hypermigration of malignant glioma cells. Radiat Oncol 6: 132, 2011.

7. Shi Q, Hjelmeland AB, Keir ST, Song L, Wickman S, Jackson D, Ohmori O, Bigner DD, Friedman HS and Rich JN: A novel lowmolecular weight inhibitor of focal adhesion kinase, TAE226, inhibits glioma growth. Mol Carcinog 46: 488-496, 2007.

8. Yang M, Li Y, Chilukuri K, Brady OA, Boulos MI, Kappes JC and Galileo DS: L1 stimulation of human glioma cell motility correlates with FAK activation. J Neurooncol 105: 27-44, 2011.

9. Reardon DA, Nabors LB, Stupp R and Mikkelsen T: Cilengitide: an integrin-targeting arginine-glycine-aspartic acid peptide with promising activity for glioblastoma multiforme. Expert Opin Investig Drugs 17: 1225-1235, 2008.

10. Nabors LB, Mikkelsen T, Hegi ME, Ye X, Batchelor T, Lesser G, Peereboom D, Rosenfeld MR, Olsen J, Brem S, Fisher JD and Grossman SA: A safety run-in and randomized phase 2 study of cilengitide combined with chemoradiation for newly diagnosed glioblastoma (NABTT 0306). Cancer 118: 5601-5607, 2012.

11. McLean GW, Carragher NO, Avizienyte E, Evans J, Brunton VG and Frame MC: The role of focal-adhesion kinase in cancer a new therapeutic opportunity. Nat Rev Cancer 5: 505-515, 2005.

12. Zhao J and Guan JL: Signal transduction by focal adhesion kinase in cancer. Cancer Metastasis Rev 28: 35-49, 2009.

13. Cance WG, Harris JE, Iacocca MV, Roche E, Yang X, Chang J, Simkins $\mathrm{S}$ and $\mathrm{Xu} \mathrm{L}$ : Immunohistochemical analyses of focal adhesion kinase expression in benign and malignant human breast and colon tissues: correlation with preinvasive and invasive phenotypes. Clin Cancer Res 6: 2417-2423, 2000.

14. Glukhova M, Koteliansky V, Sastre X and Thiery JP: Adhesion systems in normal breast and in invasive breast carcinoma. Am J Pathol 146: 706-716, 1995.

15. Gutenberg A, Brück W, Buchfelder $M$ and Ludwig HC: Expression of tyrosine kinases FAK and Pyk2 in 331 human astrocytomas. Acta Neuropathol 108: 224-230, 2004.

16. Han NM, Fleming RY, Curley SA and Gallick GE: Overexpression of focal adhesion kinase (p125FAK) in human colorectal carcinoma liver metastases: independence from c-src or c-yes activation. Ann Surg Oncol 4: 264-268, 1997.

17. Hsu NY, Chen CY, Hsu CP, Lin TY, Chou MC, Chiou SH and Chow KC: Prognostic significance of expression of nm23-H1 and focal adhesion kinase in non-small cell lung cancer. Oncol Rep 18: 81-85, 2007.

18. Kornberg LJ: Focal adhesion kinase expression in oral cancers. Head Neck 20: 634-639, 1998.

19. Owens LV, Xu L, Craven RJ, Dent GA, Weiner TM, Kornberg L, Liu ET and Cance WG: Overexpression of the focal adhesion kinase $(\mathrm{p} 125 \mathrm{FAK})$ in invasive human tumors. Cancer Res 55: 2752-2755, 1995.

20. Cordes N, Frick S, Brunner TB, Pilarsky C, Grützmann R, Sipos B, Kloppel G, McKenna WG and Bernhard EJ: Human pancreatic tumor cells are sensitized to ionizing radiation by knockdown of caveolin-1. Oncogene 26: 6851-6862, 2007.

21. Eke I and Cordes N: Dual targeting of EGFR and focal adhesion kinase in 3D grown HNSCC cell cultures. Radiother Oncol 99: 279-286, 2011.

22. Hehlgans S, Eke I and Cordes N: Targeting FAK radiosensitizes 3-dimensional grown human HNSCC cells through reduced Akt1 and MEK1/2 signaling. Int J Radiat Oncol Biol Phys 83: e669-e676, 2012.

23. Hehlgans S, Lange I, Eke I and Cordes N: 3D cell cultures of human head and neck squamous cell carcinoma cells are radiosensitized by the focal adhesion kinase inhibitor TAE226. Radiother Oncol 92: 371-378, 2009.

24. Wu ZM, Yuan XH, Jiang PC, Li ZQ and Wu T: Antisense oligonucleodes targeting the focal adhesion kinase inhibit proliferation, induce apoptosis and cooperate with cytotoxic drugs in human glioma cells. J Neurooncol 77: 117-123, 2006.

25. Storch K, Eke I, Borgmann K, Krause M, Richter C, Becker K, Schröck E and Cordes N: Three-dimensional cell growth confers radioresistance by chromatin density modification. Cancer Res 70: 3925-3934, 2010. 
26. Günther HS, Schmidt NO, Phillips HS, Kemming D Kharbanda S, Soriano R, Modrusan Z, Meissner H, Westphal M and Lamszus K: Glioblastoma-derived stem cell-enriched cultures form distinct subgroups according to molecular and phenotypic criteria. Oncogene 27: 2897-2909, 2008.

27. Wang ZG, Fukazawa T, Nishikawa T, Watanabe N, Sakurama K, Motoki T, Takaoka M, Hatakeyama S, Omori O, Ohara T, Tanabe S, Fujiwara Y, Shirakawa Y, Yamatsuji T, Tanaka N and Naomoto Y: TAE226, a dual inhibitor for FAK and IGF-IR, has inhibitory effects on mTOR signaling in esophageal cancer cells. Oncol Rep 20: 1473-1477, 2008.

28. Beierle EA, Trujillo A, Nagaram A, Golubovskaya VM, Cance WG and Kurenova EV: TAE226 inhibits human neuroblastoma cell survival. Cancer Invest 26: 145-151, 2008.
29. Watanabe N, Takaoka M, Sakurama K, Tomono Y,Hatakeyama S, Ohmori O, Motoki T, Shirakawa Y, Yamatsuji T, Haisa M, Matsuoka J, Beer DG, Nagatsuka H, Tanaka N and Naomoto Y: Dual tyrosine kinase inhibitor for focal adhesion kinase and insulin-like growth factor-I receptor exhibits anticancer effect in esophageal adenocarcinoma in vitro and in vivo. Clin Cancer Res 14: 4631-4639, 2008.

30. Golubovskaya VM, Virnig C and Cance WG: TAE226-induced apoptosis in breast cancer cells with overexpressed Src or EGFR. Mol Carcinog 47: 222-234, 2008. 